\title{
Erratum to: Comparison of hemostatic dressings for superficial wounds using a new spectrophotometric coagulation assay
}

\author{
Julian-Dario Rembe ${ }^{1 * \dagger}$, Julia K. Böhm ${ }^{1 \dagger}$, Carolin Fromm-Dornieden ${ }^{1}$, Nadine Schäfer ${ }^{1}$, Marc Maegele ${ }^{2}$, \\ Matthias Fröhlich ${ }^{2}$ and Ewa K. Stuermer ${ }^{1}$
}

\section{Erratum to: J Transl Med (2015) 13:375 DOI 10.1186/s12967-015-0740-5}

After publication of our work [1] it was brought to our attention that the cited mechanism of action for the kaolin-impregnated gauze dressing QuikClot ${ }^{\circledR}$ needs to be extended. The statement regarding the products mechanism of action was based on the most recent publication regarding QuikClot ${ }^{\circledR}$ (Reference \#20 in the article, Li et al. 2013), yet this article investigated an outdated formulation of the product based on zeolite instead of kaolin. The kaolin-impregnated gauze activates factor XII, initiating the intrinsic coagulation pathway [2]. Therefore, the following corrections are made:

1. In "Table 1 - Investigated dressings" the mechanism of action reading: "Unknown; presumably concentration of procoagulant factors due to water absorption and $\mathrm{Ca}^{2+}$ release [20]" for QuikClot ${ }^{\circledR}$ needs to be replaced with: "Activation of coagulation factor XII [20]", whereby the reference Lamb et al. 2012 (here [2]) replaces \#20 (Li et al. 2013) as reference for the mechanism of action.

2. In the discussion section page 7 of 10 , line 15 the statement "... that advances coagulation by rapidly absorbing fluid." needs to be extended by: "... and activates factor XII of the coagulation cascade."
Corrections and extension of the above statements regarding the mechanism of action of QuikClot ${ }^{\circledR}$ does not alter the findings or conclusions of the original article.

\section{Author details}

${ }^{1}$ Institute for Research in Operative Medicine (IFOM), Witten/Herdecke University, Ostmerheimer Str. 200, 51109 Cologne, Germany. ${ }^{2}$ Department of Traumatology, Orthopaedic Surgery and Sports Traumatology, Cologne-Merheim Medical Centre (CMMC), Witten/Herdecke University, Campus Cologne-Merheim, Ostmerheimer Str. 200, 51109 Cologne, Germany.

The online version of the original article can be found under doi:10.1186/s12967-015-0740-5.

Published online: 25 July 2016

References

1. Rembe J-D, Böhm JK, Fromm-Dornieden C, Schäfer N, Maegele M, Fröhlich M, Stuermer EK. Comparison of hemostatic dressings for superficial wounds using a new spectrophotometric coagulation assay. J Transl Med. 2015;30(13):375. doi:10.1186/s12967-015-0740-5.

2. Lamb KM, Pitcher HT, Cavarocchi NC, Hirose H. Vascular site hemostasis in percutaneous extracorporeal membrane oxygenation therapy. Open Cardiovasc Thorac Surg J. 2012;5:8-10.

*Correspondence: julian-dario.rembe@uni-wh.de

†Julian-Dario Rembe and Julia K. Böhm contributed equally to this work

1 Institute for Research in Operative Medicine (IFOM), Witten/Herdecke University, Ostmerheimer Str. 200, 51109 Cologne, Germany

Full list of author information is available at the end of the article 